\title{
Erratum to: Determinants of bone mineral density in young Australian women; results from the Safe-D study
}

\author{
E. T. Callegari ${ }^{1}$ - S. M. Garland ${ }^{2,3,4}$ - A. Gorelik ${ }^{5}$ - J. D. Wark Wa $^{1,6}$
}

Published online: 6 September 2017

(C) International Osteoporosis Foundation and National Osteoporosis Foundation 2017

\section{Erratum to: Osteoporos Int.}

https://doi.org/10.1007/s00198-017-4100-6

Due to an error in typesetting, in the side headings of Table 2 the sub-heading "White" under "Ethnicity" was incorrectly changed to "Yes".

The corrected table is reproduced here.

The online version of the original article can be found under https://doi. org/10.1007/s00198-017-4100-6

\section{J. D. Wark}

jdwark@unimelb.edu.au

1 Department of Medicine, Royal Melbourne Hospital, The University of Melbourne, Level 4, Clinical Sciences Building,

Parkville, VIC 3050, Australia

2 Murdoch Childrens Research Institute, Parkville, VIC, Australia

3 Royal Women's Hospital, Parkville, VIC, Australia

4 Department of Obstetrics and Gynaecology, The University of Melbourne, Parkville, VIC, Australia

5 Melbourne EpiCentre, Royal Melbourne Hospital, The University of Melbourne, Parkville, VIC, Australia

6 Bone and Mineral Medicine, Royal Melbourne Hospital, Parkville, VIC, Australia 
Table 2 Demographic and lifestyle determinants of DXA bone mineral measures in young women

\begin{tabular}{|c|c|c|c|c|c|}
\hline & Number & $\begin{array}{l}\text { Whole body } \\
\text { BMC less head } \\
\text { (g) }\end{array}$ & $\begin{array}{l}\text { Lumbar } \\
\text { spine } \\
\mathrm{aBMD}^{\mathrm{a}} \\
\left(\mathrm{g} / \mathrm{cm}^{2}\right)\end{array}$ & $\begin{array}{l}\text { Total hip } \\
\text { aBMD } \\
\left(\mathrm{g} / \mathrm{cm}^{2}\right)\end{array}$ & $\begin{array}{l}\text { Femoral neck } \\
\text { aBMD }\left(\mathrm{g} / \mathrm{cm}^{2}\right)\end{array}$ \\
\hline \multicolumn{6}{|l|}{ Demographic variables } \\
\hline \multicolumn{6}{|l|}{ Country of birth } \\
\hline Overseas & 52 & $1415 \pm 212$ & $1.00 \pm 0.11$ & $0.87 \pm 0.11$ & $0.80 \pm 0.11$ \\
\hline Australia & 274 & $1548 \pm 235$ & $1.05 \pm 0.13$ & $0.94 \pm 0.12$ & $0.87 \pm 0.13$ \\
\hline$p$ value & & $<0.001 *$ & $0.007 *$ & $<0.001 *$ & $<0.001 *$ \\
\hline \multicolumn{6}{|l|}{ Ethnicity } \\
\hline White & 273 & $1553 \pm 225$ & $1.05 \pm 0.13$ & $0.94 \pm 0.12$ & $0.88 \pm 0.13$ \\
\hline $\begin{array}{l}\text { Chinese, Japanese, } \\
\text { and Southeast } \\
\text { Asian }\end{array}$ & 38 & $1378 \pm 205$ & $1.01 \pm 0.14$ & $0.86 \pm 0.13$ & $0.79 \pm 0.12$ \\
\hline Southern Asian & 11 & $1399 \pm 364$ & $0.99 \pm 0.16$ & $0.88 \pm 0.16$ & $0.86 \pm 0.15$ \\
\hline Other & 4 & $1619 \pm 258$ & $1.04 \pm 0.08$ & $0.99 \pm 0.11$ & $0.94 \pm 0.13$ \\
\hline$p$ value & & $<0.001^{*}$ & 0.302 & $0.001 *$ & $<0.001 *$ \\
\hline \multicolumn{6}{|l|}{ White ethnicity } \\
\hline Yes & 273 & $1553 \pm 225$ & $1.05 \pm 0.13$ & $0.94 \pm 0.12$ & $0.88 \pm 0.13$ \\
\hline No & 53 & $1402 \pm 252$ & $1.00 \pm 0.13$ & $0.88 \pm 0.14$ & $0.81 \pm 0.13$ \\
\hline$p$ value & & $<0.001^{*}$ & 0.021 & $<0.001^{*}$ & $0.001 *$ \\
\hline \multicolumn{6}{|l|}{ Lifestyle variables } \\
\hline \multicolumn{6}{|l|}{ Calcium intake } \\
\hline$<1000 \mathrm{mg} /$ day & 217 & $1513 \pm 239$ & $1.03 \pm 0.11$ & $0.92 \pm 0.12$ & $0.86 \pm 0.14$ \\
\hline$\geq 1000 \mathrm{mg} /$ day & 82 & $1574 \pm 238$ & $1.06 \pm 0.16$ & $0.95 \pm 0.13$ & $0.87 \pm 0.13$ \\
\hline$p$ value & & 0.060 & 0.135 & 0.048 & 0.424 \\
\hline \multicolumn{6}{|l|}{ Physical activity } \\
\hline $\begin{array}{l}\text { Moderate-to-high } \\
\text { levels }\end{array}$ & 233 & $1543 \pm 236$ & $1.05 \pm 0.13$ & $0.94 \pm 0.12$ & $0.88 \pm 0.14$ \\
\hline $\begin{array}{l}\text { Minimal-to-low } \\
\text { levels }\end{array}$ & 91 & $1521 \pm 214$ & $1.02 \pm 0.11$ & $0.91 \pm 0.13$ & $0.84 \pm 0.12$ \\
\hline$p$ value & & 0.046 & 0.158 & 0.018 & 0.037 \\
\hline \multicolumn{6}{|l|}{ Fitness trainer use } \\
\hline No & 248 & $1508 \pm 226$ & $1.03 \pm 0.11$ & $0.92 \pm 0.12$ & $0.86 \pm 0.13$ \\
\hline $\begin{array}{l}\text { Yes - at least once } \\
\text { in previous } \\
12 \text { months }\end{array}$ & 79 & $1592 \pm 256$ & $1.07 \pm 0.16$ & $0.96 \pm 0.13$ & $0.89 \pm 0.13$ \\
\hline$p$ value & & $0.009 *$ & $0.006^{*}$ & 0.041 & 0.034 \\
\hline
\end{tabular}

Data may not add up to 326 due to incomplete survey data. Data presented as mean \pm standard deviation. Student' $\mathrm{s} t$ test or ANOVA used to test differences between categorical groups

$a B M D$ one mineral density, $B M C$ bone mineral content, $D X A$ dual-energy X-ray absorptiometry

${ }^{a}$ Twenty-two whole body scans and one lumbar spine scan were removed from analysis due to metal objects on the scan (e.g., orthopedic metal plate/screws, jewelry that could not be removed etc.)

* Denotes statistical significance after Bonferroni adjustment for multiple comparisons $(p<0.0125$ for DXA variables) 